\title{
Study on fatigue and energy-dissipation properties of nanolayered $\mathrm{Cu} / \mathrm{Nb}$ thin films
}

\author{
Y.-C. Wang, T. Hoechbauer, J. G. Swadener, T. Darling, A. Misra, \\ R. Hoagland \& M. Nastasi \\ Centre for Integrated Nanotechnologies Group, Materials Science and \\ Technology Division, Los Alamos National Laboratory, Los Alamos, USA
}

\begin{abstract}
Energy dissipation and fatigue properties of nano-layered thin films are less well studied than bulk properties. Existing experimental methods for studying energy dissipation properties, typically using magnetic interaction as a driving force at different frequencies and a laser-based deformation measurement system, are difficult to apply to two-dimensional materials. We propose a novel experimental method to perform dynamic testing on thin-film materials by driving a cantilever specimen at its fixed end with a bimorph piezoelectric actuator and monitoring the displacements of the specimen and the actuator with a fibre-optic system. Upon vibration, the specimen is greatly affected by its inertia, and behaves as a cantilever beam under base excitation in translation. At resonance, this method resembles the vibrating reed method conventionally used in the viscoelasticity community. The loss tangent is obtained from both the width of a resonance peak and a free-decay process. As for fatigue measurement, we implement a control algorithm into LabView to maintain maximum displacement of the specimen during the course of the experiment. The fatigue S-N curves are obtained.
\end{abstract}

Keywords: nanolayered thin films, fatigue, loss tangent.

\section{Introduction}

Materials consisting of nano-scale microstructures are of scientific and industrial interest due to their unusual mechanical properties. It has been shown that nanolayered multilayers exhibit ultra-high yield strength [1]. Transmission electron microscopy (TEM) studies on cold-rolled $\mathrm{Cu} / \mathrm{Nb}$ multi-layers showed that the 
films exhibit large plastic deformation without the formation of dislocation cell structures [2]. Based on the high tensile strength and lack of dislocation cell structure formation during monotonic loading, we hypothesize that the fatigue strength and failure mechanisms in nanolayered metals may differ significantly from bulk metals. However, the fatigue and energy-dissipation properties of selfsupported nano-layered thin films have not been well studied.

Experimental methods for measuring mechanical fatigue properties of materials have been well documented in many textbooks [3, 4]. In recent years, new methods are continuously under development, such as the methods for the fatigue investigations in gigacycle regime [5]. Similarly, there is a need to develop methods to evaluate fatigue properties of novel nanoscale thin film materials. We develop a method to perform fatigue tests on self-supported films via oscillating cantilever beams at their resonant frequencies. This method is superior to traditional methods, such as the tension-tension and rotating beam fatigue methods, in that high stress and high cycle fatigue can be accomplished simultaneously with fewer alignment problems. Our method applies fully reversed stress to specimens, i.e. the load ratio $\sigma_{\min } / \sigma_{\max }=-1$. Furthermore, our resonant frequency device also facilitates measurement of linear viscoelastic damping.

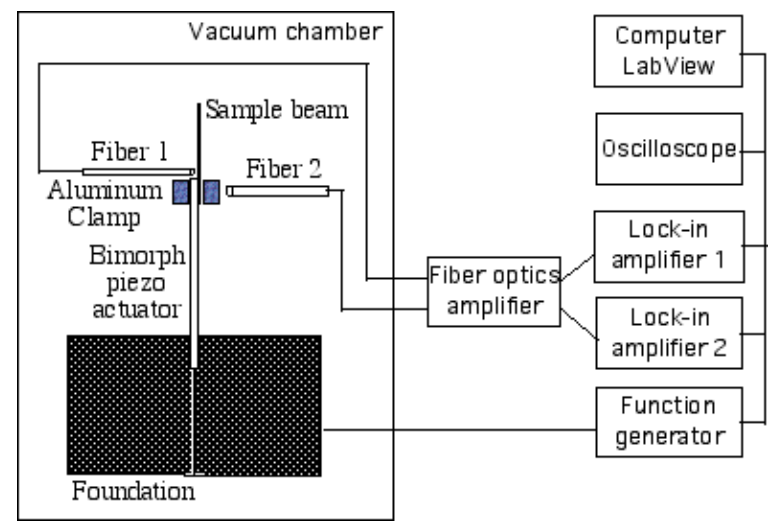

Figure 1: Schematic of the resonant frequency device for the measurement of fatigue and energy-dissipation properties of self-supported thin films.

\section{Experimental}

The schematic of our resonant frequency setup [6] is shown in Figure 1. A bimorph piezoelectric plate-like actuator (Moran Electro Ceramics, Fairfield, NJ, U.S.A.) with the dimensions of $15 \mathrm{~mm}$ in length, $6 \mathrm{~mm}$ in width and $0.64 \mathrm{~mm}$ in thickness was used to generate excitation at the support of a cantilever beam specimen. The bimorph actuator was clamped into the foundation with proper electrical connections, and driven by a function generator (Agilent 33250A, 
Agilent Technologies, Inc., Loveland, Colorado, U.S.A.). The free length of the piezo actuator was $10 \mathrm{~mm}$. The multilayer specimens were mounted onto the top of the piezo actuator with a set of aluminium clamp. The weight of the aluminium clamp was $235.4 \mathrm{mg}$. The deflection of the specimen and aluminium clamp was monitored by a fibre-optic measurement system (MTI-2000 Fotonic $^{\mathrm{TM}}$ sensor, MTI Instruments, Inc., Albany, NY, U.S.A.) with the probes labelled Fibre 1 and Fibre 2 in the figure. The location of Fibre 1 was chosen to be close to the fixed end of the specimen to avoid signal overload due to large deflection. Lock-in amplifiers (Model SR830, Stanford Research Systems, Inc., Sunnyvale, CA, U.S.A.) were connected with the fibre-optic probes to reduce noise in the displacement signals. Experiments were performed under moderate vacuum conditions typically about 50 mtorr to reduce air damping and achieve a reasonably high $\mathrm{Q}$ factor. Since our primary interest is to observe the initiation of fatigue cracks, not crack propagation, we define fatigue failure when a $10 \mathrm{Hertz}$ reduction in specimen's resonant frequency is observed.

$\mathrm{Cu} / \mathrm{Nb}$ multilayers, with individual layer thickness of $40 \mathrm{~nm}$ and consisting of 500 bilayers of $\mathrm{Cu}$ and $\mathrm{Nb}$ with a total thickness of $40 \mu \mathrm{m}$, were prepared by magnetron sputtering deposition on silicon wafers that were cut about a half way through thickness by a wafer saw into a pattern of $2 \mathrm{~mm}$ long and $1 \mathrm{~mm}$ (or 0.5 $\mathrm{mm}$ ) wide rectangular strips on the wafer surface. A typical cross-sectional TEM image of the $\mathrm{Cu} / \mathrm{Nb}$ multi-layers is shown in Figure 2 along with selected area diffraction pattern. Note the polycrystalline structure of the layer with in-plane grain size on the order of the layer thickness. The multi-layer exhibited a strong Kurdjumov-Sachs orientation relationship: $\{111\} \mathrm{Cu} / /\{110\} \mathrm{Nb}$, and $<110>\mathrm{Cu}$ $/ /<111>\mathrm{Nb}$. The parallel $\{111\} \mathrm{Cu}$ and $\{110\} \mathrm{Nb}$ planes formed the interface.

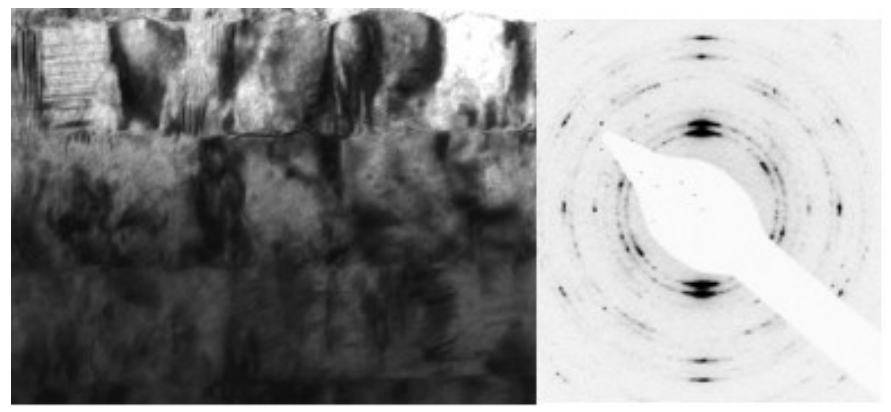

Figure 2: Cross-sectional TEM micrograph (left) and the corresponding selected area diffraction pattern (right) of a sputter-deposited $\mathrm{Cu} / \mathrm{Nb}$ multilayer with $40 \mathrm{~nm}$ individual layer thickness.

\section{Results and discussion}

It is known that the $\mathrm{Cu}$ and $\mathrm{Nb}$ films manufactured by the magnetron sputtering technique respectively have $<111>$ and $<110>$ textures parallel to their growth direction (i.e. normal to layer surfaces). However, their in-plane properties are 
composed of poly-crystals in random directions. The in-plane Young's modulus (E) of a material along a direction can be calculated as follows [7].

$$
\frac{1}{E}=\frac{\mathrm{I}^{4}}{E_{1}}+\frac{m^{4}}{E_{2}}+\frac{n^{4}}{E_{3}}+\frac{2 m^{2} n^{2}}{F_{1}}+\frac{2 \mathrm{I}^{2} n^{2}}{F_{2}}+\frac{2 m^{2} \mathrm{I}^{2}}{F_{3}},
$$

where

$$
\begin{aligned}
& \frac{1}{E_{1}}=\frac{C_{22} C_{33}-C_{23}^{2}}{\Delta}, \frac{1}{E_{2}}=\frac{C_{11} C_{33}-C_{13}^{2}}{\Delta}, \frac{1}{E_{3}}=\frac{C_{11} C_{22}-C_{12}^{2}}{\Delta}, \\
& \frac{2}{F_{1}}=\frac{2\left(C_{12} C_{13}-C_{11} C_{23}\right)}{\Delta}+\frac{1}{C_{44}}, \\
& \frac{2}{F_{2}}=\frac{2\left(C_{13} C_{23}-C_{33} C_{12}\right)}{\Delta}+\frac{1}{C_{55}}, \\
& \frac{2}{F_{3}}=\frac{2\left(C_{12} C_{23}-C_{22} C_{13}\right)}{\Delta}+\frac{1}{C_{66}}, \\
& \Delta=\left|\begin{array}{lll}
C_{11} & C_{12} & C_{13} \\
C_{12} & C_{22} & C_{23} \\
C_{13} & C_{23} & C_{33}
\end{array}\right| .
\end{aligned}
$$

Here $\mathrm{C}_{\mathrm{ij}}$ represents elastic constants and $(\ell, \mathrm{m}, \mathrm{n})$ is the direction cosine.

For single crystal $\mathrm{Cu}, \mathrm{C}_{11}=169.8 \mathrm{GPa}, \mathrm{C}_{12}=122.6 \mathrm{GPa}$ and $\mathrm{C}_{44}=75.3 \mathrm{GPa}$ [8]. The calculated principal Young's moduli are $\mathrm{E}_{1}=\mathrm{E}_{2}=\mathrm{E}_{3}=66.99 \mathrm{GPa}$. The Young modulus along $<111>$ is $\mathrm{E}_{<111>}=191.2 \mathrm{GPa}$. As for single crystal $\mathrm{Nb}, \mathrm{C}_{11}$ $=245.6 \mathrm{GPa}, \mathrm{C}_{12}=138.7 \mathrm{GPa}, \mathrm{C}_{44}=29.3 \mathrm{GPa}$ [8]. It is found that $\mathrm{E}_{1}=\mathrm{E}_{2}=\mathrm{E}_{3}$ $=145.48 \mathrm{GPa}$ and $\mathrm{E}_{<110>}=93.2 \mathrm{GPa}$ and $\mathrm{E}_{<111>}=83.2 \mathrm{GPa}$.

It can be seen from Figure 3 (a), the theoretical results of the in-plane Young's modulus of single crystalline $\mathrm{Cu}$ and $\mathrm{Nb}$, that $\mathrm{Cu}$ in (111) plane is transversely isotropic but $\mathrm{Nb}$ in (110) is direction-dependent. A simple uniform homogenization for $\mathrm{Nb}$ along different directions results in an averaged in-plane Young's modulus about $100 \mathrm{GPa}$. Since our multilayers have equal volume fraction for $\mathrm{Cu}$ and $\mathrm{Nb}$ throughout thickness, we adopt the Voigt homogenization procedure, and obtain the homogenised in-plane Young's modulus to be 115 GPa. Figure 3 (b) shows comparison between experimental and calculated inplane Young's modulus. Good agreement is achieved.

A typical SEM picture of the fatigue crack observed on the $\mathrm{Cu} / \mathrm{Nb}$ multilayers before complete fracture is shown in Figure 4 (a). The length of the sample was $7.1 \mathrm{~mm}$, and the crack caused an about $60 \mathrm{~Hz}$ reduction in resonant frequency. This sample was cycled at the stress amplitude $800 \mathrm{MPa}$ for 8500 cycles. The position of the crack is very near the aluminium clamp. The bright spots are the cyanoacrylate glue used to pre-mount the specimen on the piezo 
actuator before mounting the aluminium clamp and fatigue testing. Fatigue damage such as extrusions and intrusions are not observed around the crack on the surface where maximum stress was exerted. A SEM image of annealed $\mathrm{Cu}$ fatigued by the resonant frequency device is shown in Figure 4 (b). This sample was fatigue tested at a stress after several thousand cycles. It can be seen that two cracks were initiated at the edges of specimen, and propagated inwards. The bright regions along the cracks are slip-related intrusions and extrusions.

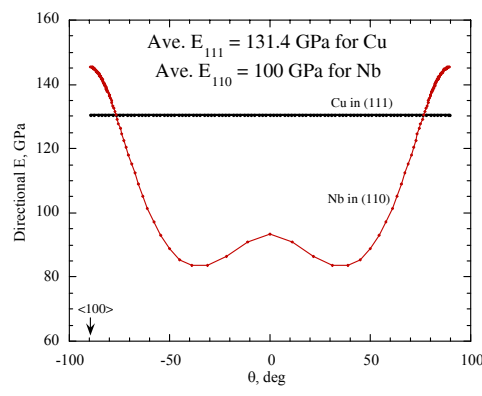

(a)

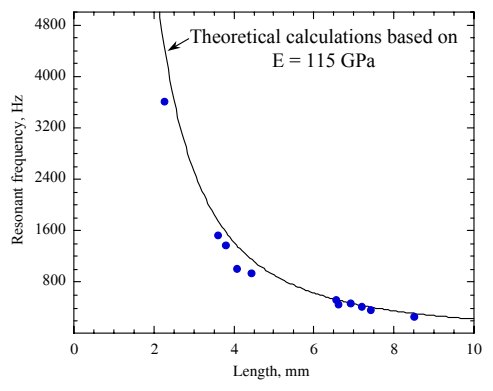

Figure 3: (a) Theoretical calculation of directional in-plane Young's modulus of the $\mathrm{Cu}$ and $\mathrm{Nb}$ with the texture of $<111>$ and $<110>$ directions normal to the layer surface, respectively, and (b) experimental results of resonant frequencies versus sample lengths.
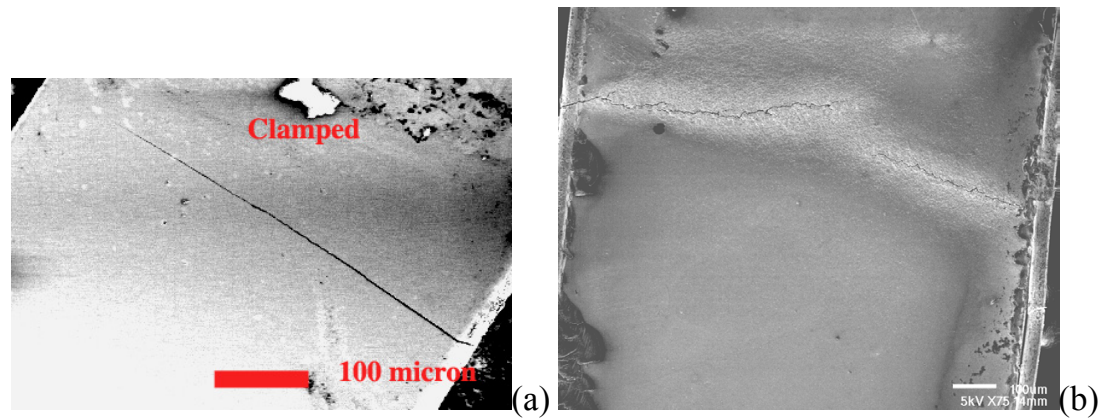

Figure 4: $\quad$ SEM images of (a) fatigued $40 \mathrm{~nm} \mathrm{Cu} / \mathrm{Nb}$ multilayers with a total thickness of $40 \mu \mathrm{m}$ and (b) fatigued annealed $40 \mu \mathrm{m}$-thick $\mathrm{Cu}$, clamped at the top of the image.

The S-N curves of several materials, quenched and tempered $\left(550^{\circ} \mathrm{F}\right) 4340$ steel, the $40 \mathrm{~nm} \mathrm{Cu} / \mathrm{Nb}$ multilayers, mild steel, 6061-T6 and annealed $\mathrm{Cu}$, are shown in Figure 5. The nanolayered $\mathrm{Cu} / \mathrm{Nb}$ exhibits a fatigue limit at about 450 MPa. It can be seen that the high fatigue endurance of the $40 \mathrm{~nm} \mathrm{Cu} / \mathrm{Nb}$ multilayers correlates well with its high tensile strength of around $1400 \mathrm{MPa}$. We obtain the ratio of fatigue endurance limit to ultimate tensile strength to be about 0.35 for the $40 \mathrm{~nm} \mathrm{Cu} / \mathrm{Nb}$ multilayers [9]. 


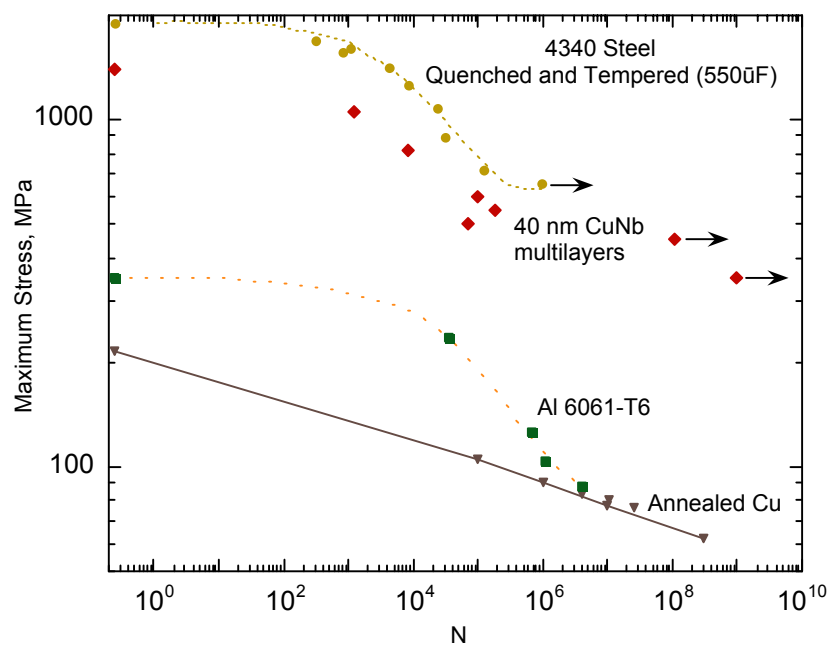

Figure 5: S-N curves (maximum stress vs. number of cycles to failure curves). Symbols are defined as follows. Circles: 4340 steel. Diamonds: 40nm Cu/nb multilayers. Triangles: mild steel. Up-side down triangles: annealed $\mathrm{Cu}$.

The deformation mechanism of the fatigue of the $40 \mathrm{~nm} \mathrm{Cu} / \mathrm{Nb}$ multilayers is not completely understood. Since the formation of dislocation cell structures is unlikely due to the nanoscale thickness of an individual layer between two adjacent interfaces, extrusion or intrusion is not observed on the surface subjected to maximum stress. This is consistent with our experimental findings. However, concerning detailed deformation mechanisms, specifically how the nanolayered thin films accumulate damage during cycling loading that eventually leads to fatigue crack initiation require further TEM study, which will be presented in a future article.

As for linear viscoelastic damping measurement in terms of loss tangent (i.e. $\tan \delta$ ), two approaches are adopted. From a resonant peak, one can use the FWHM method to calculate $\tan \delta$, as follows [10].

$$
\tan \delta=\frac{\Delta v}{v_{0} \sqrt{3}} .
$$

Here $v_{0}$ is the resonant frequency of the specimen and $\Delta v$ the frequency span at half maximum intensity. The other approach is to extract loss tangent from a free decay process, as follows.

$$
\tan \delta=\frac{1}{k \pi} \ln \frac{A_{0}}{A_{k}}
$$


Here $\mathrm{k}$ represents number of peaks in a time interval; $\mathrm{A}_{0}$ and $\mathrm{A}_{\mathrm{k}}$ are amplitudes respectively corresponding to the beginning and end of the time interval.

Figure 6 (a) and (b) show the results of our damping measurements on the $40 \mathrm{~nm} \mathrm{Cu} / \mathrm{Nb}$ multilayers. From resonant peaks, we obtain the loss tangent of the nanolayered thin films, which is approximately $3.8 \times 10^{-3}$ (thick solid line). After 20 million cycles of excitation under a small driving force (dotted line), $\tan \delta$ is $5.3 \times 10^{-3}$. The shift in resonant frequency, about $3 \mathrm{~Hz}$, is unlikely due to fatigue of the specimen. This resonant frequency shift may be system related, and it is the reason why the 10-Hertz rule is adopted for determination of fatigue failure. We remark that the measured loss tangent for the nanoscale films appears higher than expected. This may be due to defects inherently from the magnetron sputter deposition. Further experiments are underway to determine possible parasitic damping from the support of the cantilever or air damping.
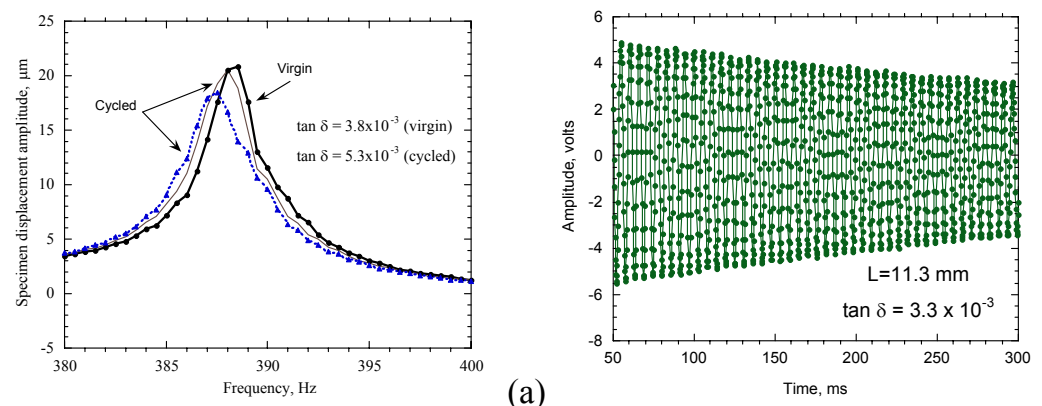

Figure 6: (a) Measured tan delta from resonant frequency peaks of a $6.5-\mathrm{mm}$ long specimen, and (b) loss tangent from the free decay method of an $11.3 \mathrm{~mm}$ long specimen. Both results were obtained under a pressure of 20 mtorr.

\section{Conclusions}

The resonant frequency method was constructed and shown to be efficient for mechanical fatigue and energy-dissipation investigations of self-supported thinfilm materials. $\mathrm{Cu} / \mathrm{Nb}$ multilayers with $40 \mathrm{~nm}$ individual layer thickness exhibit over an order of magnitude greater fatigue strength compared to bulk $\mathrm{Cu}$. No slip band intrusions and extrusions were observed near the fracture surface. The ratio of the fatigue endurance limit to ultimate tensile strength was around 0.35 , consistent with similar empirical scaling observed in other materials. Loss tangent of the nanoscale films was about $4 \times 10^{-3}$. However, potential parasitic damping from the cantilever supports requires further study.

\section{Acknowledgements}

This research was supported, in part, by the Department of Energy, Office of Science, Office of Basic Energy Sciences. YCW acknowledges support from the LANL Director's postdoctoral fellowship. 


\section{References}

[1] Clemens, B.M., Kung, H., \& Barnett, S.A. (1999). Structure and strength of multilayers. MRS Bulletin 24(2), 20-26.

[2] Misra, A., Zhang, X., Hammon, D., \& Hoagland, R.G. (2005b). Work hardening in rolled nanolayered metallic composites. Acta Materialia 53, 221-226.

[3] Frost, N.E., Marsh, K.I., \& Pook, L.P. (1974). Metal Fatigue. Clarendon Press, Oxford, UK.

[4] Suresh, S. (1998). Fatigue of Materials. Cambridge University Press, Cambridge, UK.

[5] Bathias, C., \& Paris, P.C. (2005). Gigacycle fatigue in mechanical practice. Marcel Dekker, New York.

[6] Wang, Y. C., Hoechbauer, T., Swadener, J. G., Misra, A., Hoagland, R. G., Nastasi, M., "Mechanical fatigue measurement via a vibrating cantilever beam for self-supported thin solid films", submitted (2005).

[7] Love, A.E.H. (1944). A Treatise on the Mathematical Theory of Elasticity. Dover Publications, New York.

[8] Hirth, J. P. and Lothe, J. (1982). Theory of Dislocations, Wiley, New York.

[9] Wang, Y. C., Misra, A. and Hoagland, R. G., "Fatigue properties of nanoscale $\mathrm{Cu} / \mathrm{Nb}$ multilayers", submitted (2005).

[10] Lakes, R.S. (2004). Viscoelastic measurement techniques. Review of Scientific Instruments 75(4), 797-810. 